\title{
Extending Polynomial Chaos to Include Interval Analysis
}

\author{
Antonello Monti, Senior Member, IEEE, Ferdinanda Ponci, Senior Member, IEEE, and \\ Marco Valtorta, Senior Member, IEEE
}

\begin{abstract}
Polynomial chaos theory (PCT) has been proven to be an efficient and effective way to represent and propagate uncertainty through system models and algorithms in general. In particular, PCT is a computationally efficient way to analyze and solve dynamic models under uncertainty. This paper presents a new way to use a polynomial expansion to incorporate uncertainties that are not expressed in terms of a probability density function (pdf). This paper presents the formalization of the process and some simple applications. The authors show that, within the framework introduced in this paper, it is possible to incorporate interval analysis. The long-term goal of this paper is to support the claim that the proposed framework can extract and represent uncertain behaviors in a form more general than previously used for these engineering problems. The proposed approach is first applied to an algebraic model and then to a differential equation model. The results thus obtained are analyzed in two different perspectives: 1) interpreting the PCT expansion as a fully probabilistic method and 2) in the framework of possibility theory. The conclusions in these two cases are compared and discussed.
\end{abstract}

Index Terms-Electric variable measurement, uncertainty.

\section{INTRODUCTION}

$\mathbf{R}$ EPRESENTATION and propagation of uncertainty have been discussed for many years, and different approaches have been proposed in the literature. One critical aspect of this debate is the definition of the formalism able to capture any possible source of uncertainty. In particular, an important question is to understand if the probability theory is sufficient to describe uncertain processes or if the vision should be extended to include the concept of possibility. This latter approach leads to the theory of evidence [1], [3].

The main problem that the theory of evidence tries to solve is how to treat the presence of uncertainties, whose probability density function (pdf) is not or cannot be known.

Polynomial chaos theory (PCT) was introduced and applied as a pure probabilistic method [4]. Its application to electrical engineering problems and to measurements in particular has been proposed and detailed in [5]-[9].

Manuscript received September 30, 2008; revised January 15, 2009. First published September 29, 2009; current version published December 9, 2009. This work was supported by the U.S. Office of Naval Research under Grant N00014-07-1-0603. The Associate Editor coordinating the review process for this paper was Dr. Dario Petri.

A. Monti and F. Ponci are with the E.ON Energy Research Center, RheinischWestfaelische Technische Hochschule Aachen, 52066 Aachen, Germany (e-mail: amonti@eonerc.rwth-aachen.de; fponci@eonerc.rwth-aachen.de).

M. Valtorta is with the Department of Computer Science and Engineering, University of South Carolina, Columbia, SC 29208 USA (e-mail: mgv@engr.sc.edu).

Color versions of one or more of the figures in this paper are available online at http://ieeexplore.iee.org.

Digital Object Identifier 10.1109/TIM.2009.2025688
The purpose of this paper is to propose a new polynomial expansion of uncertain variables to extract, from the analysis of the variable, the same information extracted with the application of interval analysis and then of the theory of evidence. This concept was first proposed in [1], whereas in this paper, a more complete comparison with the approach of the theory of evidence, including the analysis of the dynamic model, is presented.

The results presented here show that, with PCT, it is possible to define the variable in such a way that the uncertainty can be propagated, as proposed in [10] and [11] with the theory of evidence. The possible elements of connection with what is proposed in these reference papers are also discussed here.

This paper is organized as follows: Section II introduces the new representation of uncertainty as an extension of the PCT expansion, whereas Section III reviews the basic operations comparing interval analysis and PCT. Section IV introduces the generalized process together with an example. The results are then used in Section V for a comparison with the random fuzzy variable approach. Finally, Section VI extends the approach to a dynamic model, including differential equations providing a new example of comparison.

\section{New Polynomial Approach}

One of the major advantages of PCT is the possibility to solve, in time-domain simulation, a system of differential equations affected by parametric uncertainty with a single run of the model [12]. This is particularly important for all the cases in which the online processing of uncertainty is needed, such as in monitoring applications. Examples of these situations are discussed in [6].

The PCT approach that allows achieving this is described herein.

Let us introduce a general definition of an uncertain dynamic model: an uncertain dynamic model is here defined as a set of differential equations of the form

$$
\frac{d \bar{x}}{d t}=\bar{f}\left(\bar{x}, p_{d}, p_{p r}, p_{p o}, t\right)
$$

where

$\bar{x} \quad$ vector of the state variables of the system;

$\bar{f} \quad$ vector of functions;

$p_{d} \quad$ set of parameters that are defined with no uncertainty;

$p_{p r} \quad$ set of uncertain parameters with known pdf;

$p_{p o} \quad$ set of uncertain parameters with unknown pdf;

$t$ time variable. 
Let us now suppose that the set $p_{p r}$ has the cardinality $n_{p r}$, and the set $p_{p o}$ has the cardinality $n_{p o}$. The new polynomial expansion for a generic variable $y$ of the system is defined as

$$
y=\sum_{i=0}^{p} y_{i} \psi_{i}(\boldsymbol{\xi}, \boldsymbol{\eta})
$$

This new expansion formally looks as the standard form [4], but here, the variables have been split into the following two sets: i.e., vectors $\boldsymbol{\xi}$ and $\boldsymbol{\eta}$ as

1) $\boldsymbol{\xi}=\left\{\xi_{1}, \xi_{2}, \ldots, \xi_{n_{p r}}\right\}$, the set of variables associated to uncertainties with known pdf;

2) $\boldsymbol{\eta}=\left\{\eta_{1}, \eta_{2}, \ldots, \eta_{n_{p_{o}}}\right\}$, the set of variables associated with uncertainties with unknown pdf.

Let us now focus on the case in which all the known pdf's can optimally be expressed by means of the Legendre expansion. This choice minimizes the number of terms for a uniform distribution, whereas it requires a functional mapping for all the other cases [4]. If we perform the same expansion for the parameters with unknown pdf, we can represent a generic uncertain variable $k$ as

$$
k=k_{o}+k_{1} \eta
$$

where

$k_{o} \quad$ center of the uniform interval of uncertainty;

$k_{1} \quad$ half of the interval size.

As a result of this expansion, the evaluation of the extremes of the polynomial function represents the external values of the interval. This aspect of the problem has already been discussed in detail in [9]. Here, the authors show how this result can help in supporting interval analysis within the framework of PCT.

The same approach can obviously be applied to models expressed in terms of algebraic equations instead of differential equations.

\section{ELEMENTARY OPERATIONS IN INTERVAL ANALYSIS}

In this section, the elementary Sum and Product operations are presented as performed with interval analysis and with the equivalent process performed in a PCT expansion.

Let us consider two uncertain variables $a$ and $b$ defined by an interval and unknown pdf as

$$
\begin{aligned}
a & =\left[a_{\min }, a_{\max }\right] \\
b & =\left[b_{\min }, b_{\max }\right] .
\end{aligned}
$$

By applying (3), the following expressions are obtained:

$$
\begin{aligned}
& a=a_{o}+a_{1} \eta_{1} \\
& b=b_{0}+b_{1} \eta_{2}
\end{aligned}
$$

where

$$
\begin{aligned}
& a_{0}=\frac{a_{\text {max }}+a_{\text {min }}}{2} \\
& a_{1}=\frac{a_{\text {max }}-a_{\text {min }}}{2} \\
& b_{0}=\frac{b_{\text {max }}+b_{\text {min }}}{2} \\
& b_{1}=\frac{b_{\text {max }}-b_{\text {min }}}{2}
\end{aligned}
$$

and $\eta_{1}$ and $\eta_{2}$ are two Legendre expansion variables, as introduced in Section II.

The sum and multiplication operations between $a$ and $b$ are now introduced.

The general expression of the sum of two uncertain variables expressed in PCT form is

$$
s_{a b}=a+b=\sum a_{k} \Phi_{k}(\boldsymbol{\eta})+\sum b_{k} \Phi_{k}(\boldsymbol{\eta}) .
$$

Given the (4) and (5) form of the two uncertain variables, the calculation of (7) is trivial. Assuming, for the sake of simplicity but without loss of generality, that $a_{0}, a_{1}, b_{0}$, and $b_{1}$ are positive, and evaluating $s_{a b}$ at $\boldsymbol{\eta}_{\max }=\left[\begin{array}{l}1 \\ 1\end{array}\right]$ and $\boldsymbol{\eta}_{\min }=\left[\begin{array}{c}-1 \\ -1\end{array}\right]$, the following is obtained:

$$
\begin{aligned}
(a+b)_{\max } & =a_{0}+a_{1}+b_{o}+b_{1}=a_{\max }+b_{\max } \\
(a+b)_{\min } & =a_{0}-a_{1}+b_{o}-b_{1}=a_{\min }+b_{\min } .
\end{aligned}
$$

This result yields the same interval obtained using interval analysis algebra on $a+b$.

If the values are not all positive, then the correct maximum and minimum points will be in one of the other extreme values of the interval defined by the domain of the variables $\eta_{1}$ and $\eta_{2}$. A general solution can be obtained by simple comparison, as in standard interval analysis [13].

The general expression of the product of two uncertain variables expressed in PCT form is

$$
p_{a b}=a \cdot b=\sum a_{k} \Phi_{k}(\boldsymbol{\eta}) \cdot \sum b_{k} \Phi_{k}(\boldsymbol{\eta}) .
$$

Given the definition of the two uncertainties, the calculation of (9) is trivial. Assuming, for the sake of simplicity but without loss of generality, that $a_{0}, a_{1}, b_{0}$, and $b_{1}$ are positive, and evaluating the resulting variable in $\boldsymbol{\eta}_{\max }=\left[\begin{array}{l}1 \\ 1\end{array}\right]$ and $\boldsymbol{\eta}_{\min }=$ $\left[\begin{array}{l}-1 \\ -1\end{array}\right]$, we obtain

$$
\begin{aligned}
(a \cdot b)_{\text {max }} & =a_{0} b_{0}+a_{0} b_{1}+a_{1} b_{0}+a_{1} b_{1}=a_{\text {max }} b_{\text {max }} \\
(a \cdot b)_{\text {min }} & =a_{0} b_{0}-a_{0} b_{1}-a_{1} b_{0}+a_{1} b_{1}=a_{\text {min }} b_{\text {min }} .
\end{aligned}
$$

This result yields the same interval obtained using interval analysis algebra on $a \cdot b$.

If the values are not all positive, then the correct maximum and minimum points will be in one of the other extreme values of the interval defined by the domain of the variables $\eta_{1}$ and $\eta_{2}$. In addition, in this case, the general solution can be obtained by simple comparison, as in standard interval analysis [13].

More in general, for every elementary operation, given two intervals used as operands and defined in PCT format as in (5), and given the resulting PCT expression of the output of the operation defined as

$$
y_{a b}=\sum y_{k} \Phi_{k}(\boldsymbol{\eta})
$$

the new interval representing the output variable $y$ is defined as

$$
\begin{aligned}
y_{a b} & =\left[y_{\min }, y_{\max }\right] \\
& =\left[\min _{\eta}\left(\sum y_{k} \Phi_{k}(\boldsymbol{\eta})\right), \max _{\eta}\left(\sum y_{k} \Phi_{k}(\boldsymbol{\eta})\right)\right] .
\end{aligned}
$$

The procedures to calculate (12) are detailed in [17]. As described in the reference, the solution is trivial considering that we calculate the max and min of a polynomial function 
in an interval that is closed and limited. In the majority of the cases, the minimum and maximum are actually on the boundary of the domain. A typical example, as describe above, is when all the quantities are positives, and the max and min are at the two extrema of all $\boldsymbol{\eta}$ variables.

More practically, we can say that, given two uncertain variables, we can substitute the uncertain interval with a variable with uniform distribution. The propagation as interval always represents the worst cases of the probability function or also the interval of definition of the output variable itself. As the result of this property, we can always calculate the propagation of uncertainty using the probabilistic definition and extract the interval information as the extreme case.

\section{General Process for Uncertainty Propagation}

From the results presented in the previous section, a general process is derived to define uncertain models in which variables with known and unknown pdf may be combined. The main steps to be followed are summarized as follows:

1) For each uncertain parameter, define an uncertain variable. If the pdf of the parameter is known, then the variable belongs to the set $\boldsymbol{\xi}=\left\{\xi_{1}, \xi_{2}, \ldots, \xi_{n_{p r}}\right\}$; otherwise, it belongs to the set $\boldsymbol{\eta}=\left\{\eta_{1}, \eta_{2}, \ldots, \eta_{n_{p o}}\right\}$.

2) Propagate this variable through the model and perform the Galerkin projection [4].

3) Solve the model and obtain the output variable $y=$ $\sum_{i=0}^{p} y_{i} \psi_{i}(\boldsymbol{\xi}, \boldsymbol{\eta})$.

4) Solve the max-min problem for the set $\eta=$ $\left\{\eta_{1}, \eta_{2}, \ldots, \eta_{n_{p o}}\right\}$.

5) Apply the appropriate distribution for the set $\boldsymbol{\xi}=$ $\left\{\xi_{1}, \xi_{2}, \ldots, \xi_{n_{p r}}\right\}$.

An example of the application of these steps is herein introduced.

Let us consider the calculation of the active power in a threephase sinusoidal system. If Aron insertion is adopted, then the two power measurements are required. Assuming a digital processing operation, we consider as input of the algorithm the measurement of two currents and two voltages. The instantaneous power is defined by following the well-known formula

$$
p=v_{13} i_{1}+v_{23} i_{2} .
$$

Let us suppose that the following information is available for each of the measurements.

\section{A. Voltage Measurement}

Both voltage measurements are affected by two uncertainty contributions each: a first contribution has an unknown pdf and is defined over an interval with width of $0.1 \mathrm{~V}$, and a second contribution has a uniform pdf with interval amplitude of $0.05 \mathrm{~V}$.

\section{B. Current Measurement}

Both current measurements are affected by two uncertainty contributions each: the first uncertainty contribution has an unknown pdf and is defined over an interval with width of $0.2 \mathrm{~A}$, and the second uncertainty contribution has a uniform pdf with an interval amplitude of $0.08 \mathrm{~A}$.

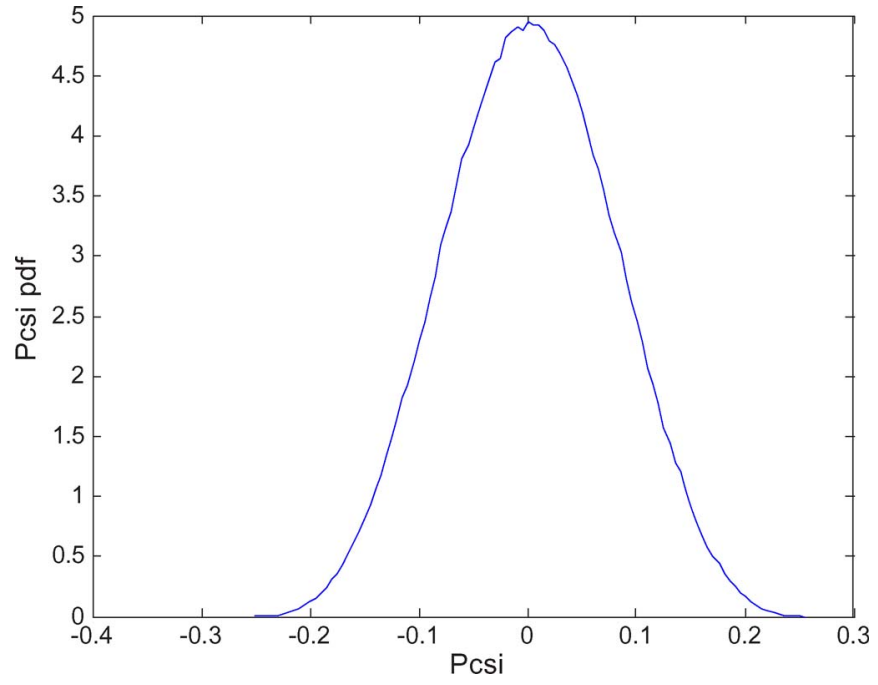

Fig. 1. PDF for the component $P_{\xi}$.

The following representation of the electrical variables can thus be introduced as

$$
\begin{aligned}
v_{13}\left(\eta_{1}, \xi_{1}\right) & =v_{130}+0.1 \eta_{1}+0.05 \xi_{1} \\
v_{23}\left(\eta_{2}, \xi_{2}\right) & =v_{230}+0.1 \eta_{2}+0.05 \xi_{2} \\
i_{1}\left(\eta_{3}, \xi_{3}\right) & =i_{10}+0.2 \eta_{3}+0.08 \xi_{3} \\
i_{2}\left(\eta_{4}, \xi_{4}\right) & =i_{20}+0.2 \eta_{4}+0.08 \xi_{4} .
\end{aligned}
$$

The total active power can be calculated by substituting (12) in (11). By applying what is described in Section III, we obtain

$$
\begin{aligned}
P= & \left(v_{130}+0.1 \eta_{1}+0.05 \xi_{1}\right)\left(i_{10}+0.2 \eta_{3}+0.08 \xi_{3}\right) \\
& +\left(v_{230}+0.1 \eta_{2}+0.05 \xi_{2}\right)\left(i_{20}+0.2 \eta_{4}+0.08 \xi_{4}\right) .
\end{aligned}
$$

Rearranging the terms, the following elements can be isolated:

$$
\begin{aligned}
P_{0}= & v_{130} i_{10}+v_{230} i_{20} \\
P_{\eta}= & i_{10} 0.1 \eta_{1}+v_{130} 0.2 \eta_{3}+0.02 \eta_{1} \eta_{3} \\
& +i_{20} 0.1 \eta_{2}+v_{230} 0.2 \eta_{4}+0.02 \eta_{2} \eta_{4} \\
P_{\xi}= & i_{10} 0.05 \xi_{1}+v_{130} 0.08 \xi_{3}+0.0004 \xi_{1} \xi_{3} \\
& +i_{20} 0.05 \xi_{2}+v_{230} 0.08 \xi_{4}+0.0004 \xi_{2} \xi_{4} \\
P_{\eta \xi}= & 0.008 \eta_{1} \xi_{3}+0.001 \eta_{3} \xi_{1}+0.008 \eta_{2} \xi_{4}+0.001 \eta_{4} \xi_{2} .
\end{aligned}
$$

Let us now individually analyze each term.

$P_{0}$ represents the central value (mean value) of the measurement.

$P_{\eta} \quad$ represents the contribution to the uncertainty only determined by elements with an unknown pdf. If all the measurements are positive, then the max problem is simply solved, and all the $\eta$ variables will be set equal to 1 , determining an interval of amplitude

$$
\bar{P}_{\eta}=i_{10} 0.1+v_{130} 0.2+i_{20} 0.1+v_{230} 0.2+0.04 .
$$

$P_{\xi} \quad$ represents the contribution to the uncertainty only determined by elements with a known pdf. Notice that the resulting pdf is no longer uniform, as reported in Fig. 1.

$P_{\eta \xi}$ represents the interaction between the uncertainties determined by a known pdf and an unknown pdf. The analysis and treatment of this component are split in two parts. A max problem is solved for the $\eta$ variables, 
thus yielding a random contribution with a known pdf. In this example, the problem has to be solved element by element for each of the $\eta$ variables.

In conclusion, we can identify two major components of the combined uncertainty: one with an unknown pdf defined by the interval $\left[P_{0}-\bar{P}_{\eta}, P_{0}+\bar{P}_{\eta}\right]$, and one with a known pdf defined by the sum of the contributions given by the terms $P_{\xi}$ and $P_{\eta \xi}$.

\section{Relation With the Random FuZzy Variables AND ALFA-CUT}

Considering the comprehensive nature of the approach proposed here, the natural term of comparison is the approach proposed in [10] and [11]. In fact, because the propagation of uncertainty in the PCT framework proposed here combines the algebra of intervals with the classical probabilistic approach, it is reasonably simple to define a direct relation between the proposed representation and a random fuzzy variable.

A fuzzy variable, in general, is defined by a set of membership functions. From the uncertainty theory standpoint, given a membership function value, this determines over a membership an interval value called alpha-cut. An alpha-cut can be seen as a confidence interval, whose confidence level is 1- $\alpha$. Fuzzy variables have been extended to the concept of random fuzzy variable, where a combination of alpha-cuts provides the confidence level related to the probabilistic part of the uncertainty and the nonprobabilistic part of the uncertainty [10], [11], [16], [18].

The analysis is limited to the case in which the uncertainty contribution with unknown pdf has the same contribution for each value of the alpha-cuts.

Under this assumption, the process of comparison follows four steps:

1) Determine the contribution given by the unknown pdf and calculate the internal interval of the alpha-cuts.

2) Perform the transformation from the PCT expansion representation of the contribution with known pdf to the probability distribution function. This can be performed either numerically, by generating random number cases to be used in the polynomial expansion, or analytically, as in [14].

3) Invert the relation to determine the alpha-cuts.

4) Combine the two intervals in a set of alpha-cuts.

By considering the same example discussed in the previous section, we report in Figs. 2-4 the fuzzy diagrams for the voltage and current variables and for the calculated total power. The calculations are performed assuming the following central values for the measurements:

$$
\begin{aligned}
\mathrm{v}_{13} & =-0.6 \mathrm{~V} \\
\mathrm{i}_{1} & =-0.4 \mathrm{~A} \\
\mathrm{v}_{23} & =1 \mathrm{~V} \\
\mathrm{i}_{2} & =1 \mathrm{~A} .
\end{aligned}
$$

The values are chosen intentionally small to make the understanding of the calculation process easier. They could alternatively be considered as per-unit values.

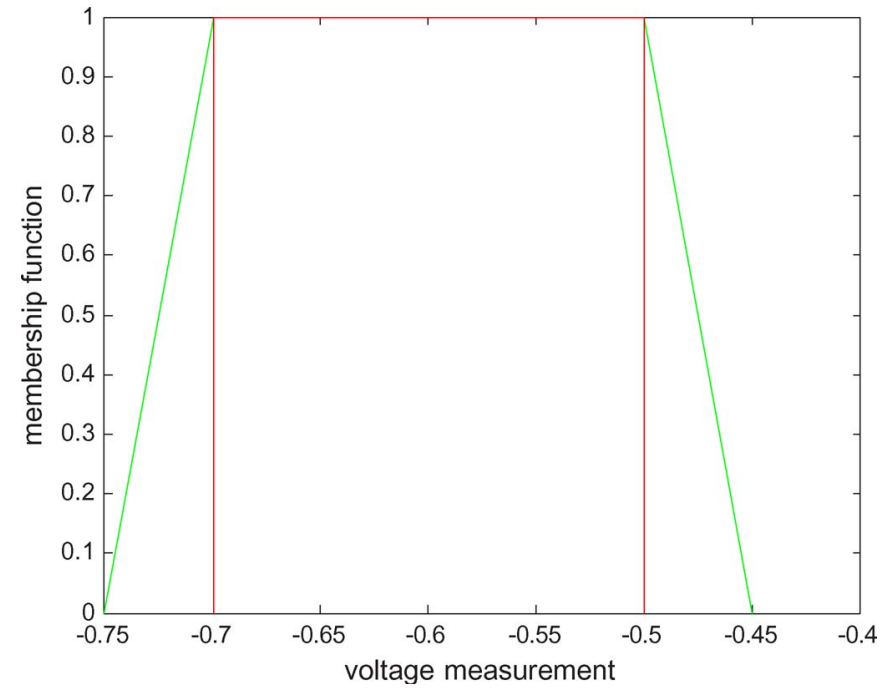

Fig. 2. RFV for the voltage measurement.

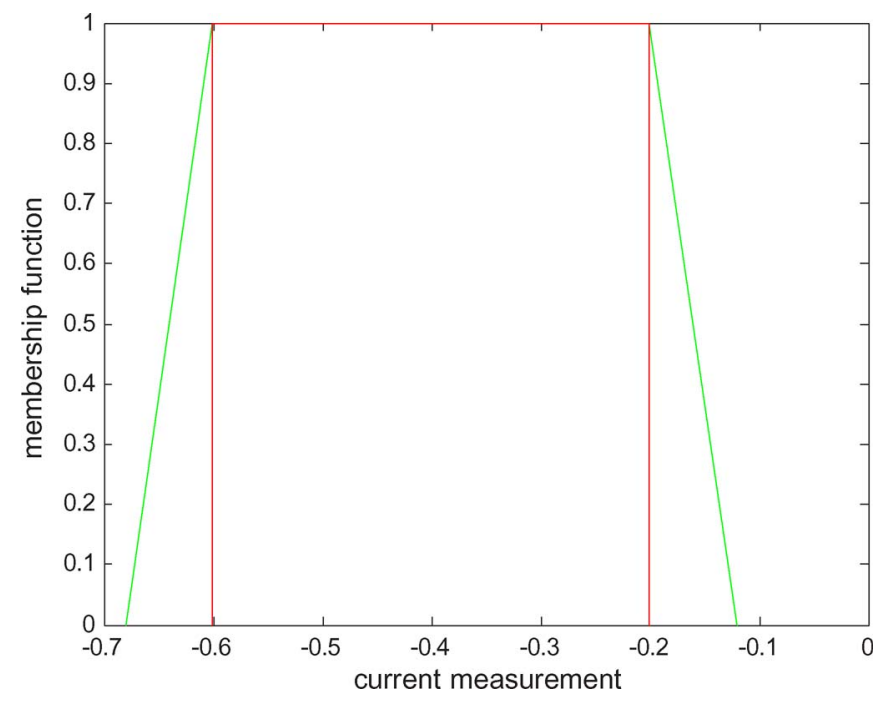

Fig. 3. RFV for the current measurement.

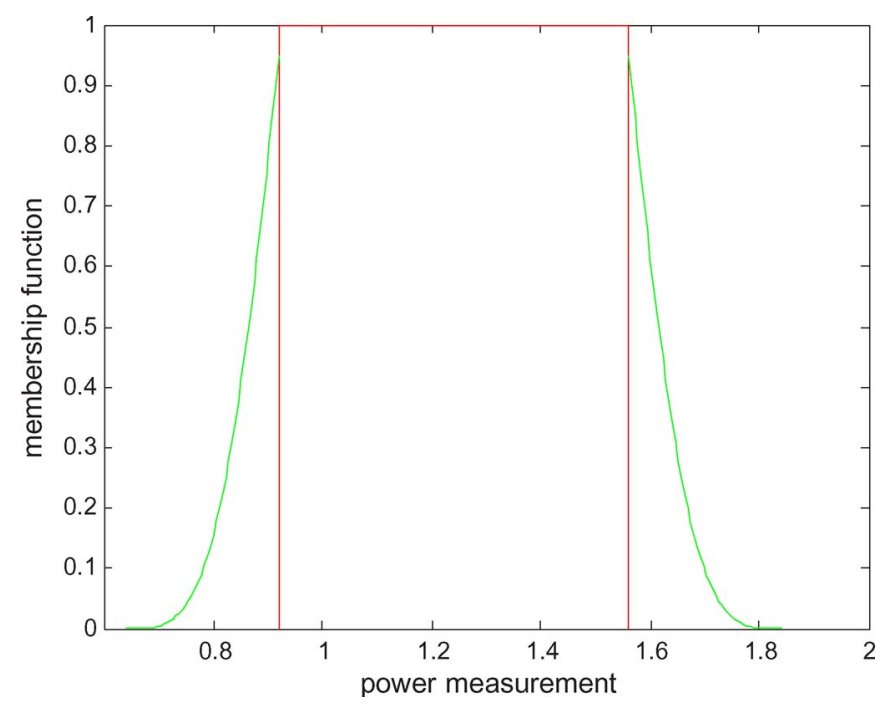

Fig. 4. RFV for the calculated power measurement. 


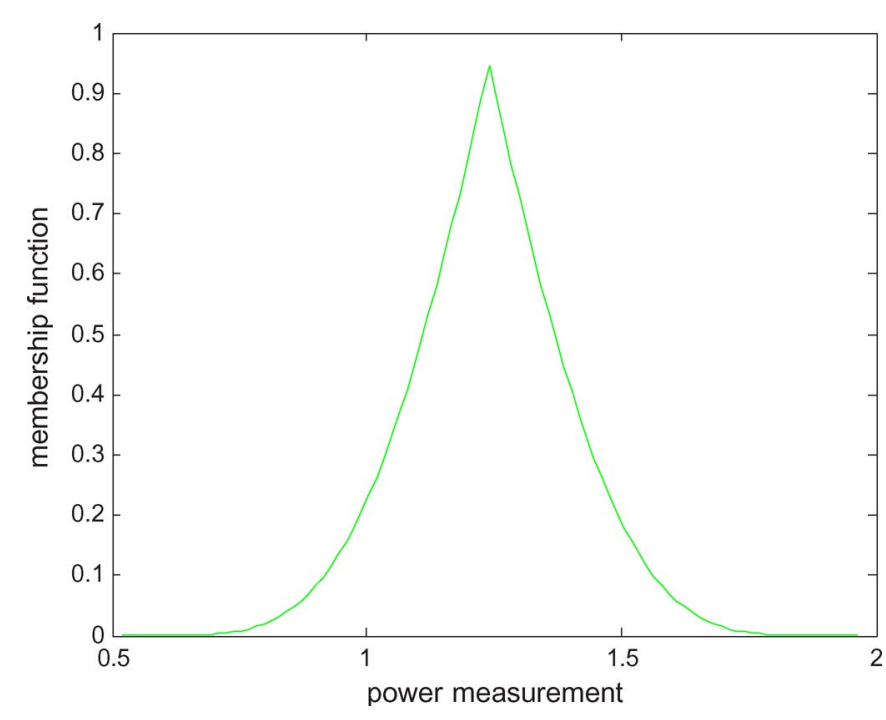

Fig. 5. RFV calculated assuming only uniform distribution.

Let us now repeat the calculation assuming a uniform distribution for all the $\eta$ variables. This case corresponds to making an assumption that whenever a pdf is not known a priori, it can be substituted with a uniform distribution. The adoption of the uniform pdf in this case is quite common as a choice to represent an unbiased approach to each of the possible values. For what concerns the PCT description, it should be noticed that (15) still holds. The difference here is the way in which we interpret the function, because we skip the step in which the max uncertainty contribution due to the $\eta$ variables is computed. Nevertheless, we can exploit the analytical nature of the PCT solution to quickly calculate the alpha-cuts.

The results are reported in Fig. 5. Notice that, consistent with the theory of evidence, the assumption of uniform distribution for the unknown pdf determines smaller intervals for the same alpha-cuts. The differences are particularly evident in this case, because the contribution with an unknown pdf is the most significant for each of the measured quantities. It is also pointed out that, since the form of the PCT solution is homogeneous, the user can easily compare the effect of one (uniform pdf) or the other (unknown pdf) assumption before deciding.

\section{UnCertainty Propagation Through DYNAMIC MODELS}

Let us now extend the application of the proposed methodology to the case of a dynamic model. This case best demonstrates the most significant advantages of the proposed PCT approach. Following what was introduced in Section II, let us consider a set of differential equations expressed in normal form as

$$
\dot{\mathbf{x}}=f(\mathbf{x}, t)
$$

where $x$ is a vector of state variables, and $f$ is a vector of functions. For the sake of simplicity, let us only focus on the linear case, where the previous equation takes the form of

$$
\dot{\mathbf{x}}=\mathbf{A x}+\mathbf{B u}
$$

with $A$ as a square matrix $n \times n$, where $n$ is the number of state variables, and $B$ as an $n \times m$ matrix, where $m$ is the number of exogenous inputs.
Depending on the application, there may only be parametric uncertainty in parameter matrices $A$ and $B$ or also in the input $u$.

Following the same approach used before, we can categorize these uncertainties in two different categories: 1) uncertainties with known pdf and 2) uncertainties with unknown pdf and known range of possibilities. Each term of the first type is associated with a variable of the $\xi$ set, and each term of the second type is associated with a variable of the $\eta$ set. As introduced in Section IV, we can expand the model and then perform a Galerkin projection. As a result, an extended set of state variables is obtained. They formally bear the same structure but with different coefficients and different order, e.g.,

$$
\dot{\mathbf{x}}_{p c t}=\mathbf{A}_{p c t} \mathbf{x}_{p c t}+\mathbf{B}_{p c t} \mathbf{u}_{p c t} .
$$

As already presented in [8], this system can be integrated with any traditional integration method, thus providing as result the time evolution of the coefficients of expansion.

In general, for each state variable of the original system, we can write

$$
x(t)=\sum_{i=0}^{p} x_{i}(t) \psi_{i}(\boldsymbol{\xi}, \boldsymbol{\eta}) .
$$

At any given instant, the previous expression can be read in the following different ways:

1) Operating in the framework of the possibility approach, we can separate the analysis of the terms in two categories: the terms function of $\eta$ are analyzed through a worst-case calculation, whereas the terms function of $\xi$ will be treated according to a uniform distribution.

2) Assuming that a uniform distribution is a good representation of the lack of knowledge, the same formula above still holds but it is to be interpreted in a different way and treating variables $\eta$ and $\xi$ in the same way.

This process has two major advantages:

1) The mapping to a PCT representation makes the integration of the model a rather easy task (assuming the number of uncertainties is reasonably limited), whereas the integration of a model in the form of random fuzzy variable may significantly be time consuming.

2) For any generic time instant, it is rather straightforward to perform the mapping of the expansion to a comprehensive pdf (full probabilistic approach) or to a random fuzzy variable (theory of evidence). The user can then compare the results and evaluate the effects of one or of the other approach in terms of confidence intervals.

\section{Dynamic Model: ExAmple of APPLICATION}

The approach proposed in the previous section is here exemplified and analyzed. Let us consider, for example, the following dynamic model of an electromechanical system:

$$
\frac{d}{d t}\left[\begin{array}{c}
i \\
\omega
\end{array}\right]=\left[\begin{array}{cc}
-R / L & k \\
k / J & H / J
\end{array}\right]\left[\begin{array}{c}
i \\
\omega
\end{array}\right]+\left[\begin{array}{cc}
1 / L & 0 \\
0 & 1 / J
\end{array}\right]\left[\begin{array}{c}
v \\
T_{l}
\end{array}\right]
$$


where

$i \quad$ armature current;

$\omega$ rotor speed;

$R$ armature resistance;

$L \quad$ armature inductance;

$k$ torque constant;

$v$ armature voltage;

$H$ drag coefficient;

$J \quad$ rotor inertia;

$\mathrm{Tl}$ external resistance torque.

This model is typically used in electrical drive application for the indirect measurement of speed [15]. The equations are written for a dc machine, but they would formally be the same for an induction machine or a brushless machine if we substitute the armature quantities with the quadrature axis quantities in the Park domain.

The main purpose of this model is estimate speed from electrical measurements. The main problem is the uncertainty in the mechanical section. While we can assume that the inertia at the shaft can be known with reasonable accuracy, the following two problems remain unresolved:

1) The drag coefficient $H$ may change in time. For this problem, we can assume that a statistical analysis can be performed, and that the changes can be mapped to a known pdf.

2) The model of the load. Modeling the load simply as a drag torque proportional to the speed does not cover most of the practical applications. For this reason, we introduced in the model an external disturbance called torque $T l$. For this term, typically, a range variation can be determined, but it is impossible to know its pdf a priori.

Following these hypotheses, we can study the impact of these two uncertainties and their propagation throughout the model. For the sake of simplicity, let us now assume that the current and voltage measurements are ideal so that the analysis is limited to the impact of the uncertainty in the indirect measurement process. The impact of the nonideality of the sensor could be integrated in a further stage, as described in [6].

The two sources of uncertainty are now formalized as follows:

1) Let us suppose that $H$ has a uniform distribution (so a variable $\xi$ will be associated to it) as

$$
H=H_{o}+H_{1} \xi
$$

2) Let us suppose that $T l$ is uncertain, and that no knowledge of the distribution is available. As a result, it will be
TABLE I

DATA FOR THE MODEL

\begin{tabular}{|c|c|}
\hline Armature resistance & $1 \Omega$ \\
\hline Armature inductance & $0.001 \mathrm{H}$ \\
\hline Inertia & $0.01 \mathrm{kgm} 2$ \\
\hline Drag coefficient & $\begin{array}{c}\text { Uniform distribution with } \\
\text { center value of } 0.3 \mathrm{Nms} \text { and } \\
\text { interval of variation of } 0.05\end{array}$ \\
\hline Resistant torque & $\begin{array}{c}\text { Interval of variation centered } \\
\text { around } 1 \mathrm{Nm} \text { with variation } \\
\text { of } 0.4 \mathrm{Nm} \text { and unknown } \\
\text { distribution }\end{array}$ \\
\hline Torque constant & $0.1 \mathrm{Nm} / \mathrm{A}$ \\
\hline Voltage at the armature & $100 \mathrm{~V}$ \\
\hline
\end{tabular}

associated with a variable of type $\eta$ as

$$
T_{l}=T_{l o}+T_{l 1} \eta
$$

Performing the Galerkin projection and cutting the expansion at the first order, the extended PCT new model is expressed as in (25), shown at the bottom of the page.

This model has been implemented in Matlab and integrated in time. As a result, we obtain the expression of the timedomain polynomial expansion of the speed as a function of two uncertainties.

The data reported in the following table has been used in the simulation.

The motor is started at a constant voltage with no external resistant torque. At time $t=0.5 \mathrm{~s}$, a step of resistant torque is applied with uncertain amplitude and unknown probability distribution (see Table I for the values).

The transient of the central value of the speed is illustrated in Fig. 6.

For every time instant, it is then possible to compare the impact of one approach (fully probabilistic) and the other (theory of evidence).

Let us focus on two different time instants. At time $0.5 \mathrm{~s}$, very close to the start of the new transient, the impact of the resistant torque is still limited. To appreciate this case, it is interesting to compare Figs. 7 and 8 . The random fuzzy variable (RFV) is indicating a behavior close to a triangular membership, which is a symptom of perfectly flat probabilistic distribution function and limited effect of the possibility aspect. The situation is significantly different at $t=0.7 \mathrm{~s}$, as can be seen in Figs. 9 and 10. At $t=0.7 \mathrm{~s}$, the effect of the resistant torque is significant, and while the pdf of the full probabilistic approach tends to move away from a flat distribution to become more trapezoidal, the RFV is now presenting a significant flat part, thus affecting the definition of the confidence interval.

$$
\frac{d}{d t}\left[\begin{array}{c}
i_{0} \\
i_{\eta} \\
i_{\xi} \\
\omega_{0} \\
\omega_{\eta} \\
\omega_{\xi}
\end{array}\right]=\left[\begin{array}{cccccc}
-R / L & 0 & 0 & -k t / L & 0 & 0 \\
0 & -R / L & 0 & 0 & -k t / L & 0 \\
0 & 0 & -R / L & 0 & 0 & -k t / L \\
k t / J & 0 & 0 & -H o / J & 0 & -1 / 3 H_{1} / J \\
0 & k t / J & 0 & 0 & -H o / J & 0 \\
0 & 0 & k t / J & -H_{1} / J & 0 & -H 0 / J
\end{array}\right]+\left[\begin{array}{ccc}
1 / L & 0 & 0 \\
0 & 0 & 0 \\
0 & 0 & 0 \\
0 & -1 / J & 0 \\
0 & 0 & -1 / J \\
0 & 0 & 0
\end{array}\right]\left[\begin{array}{c}
v(t) \\
T_{o}(t) \\
T_{1}(t)
\end{array}\right]
$$






Fig. 6. Time evolution of the central value according to the PCT dynamic model.

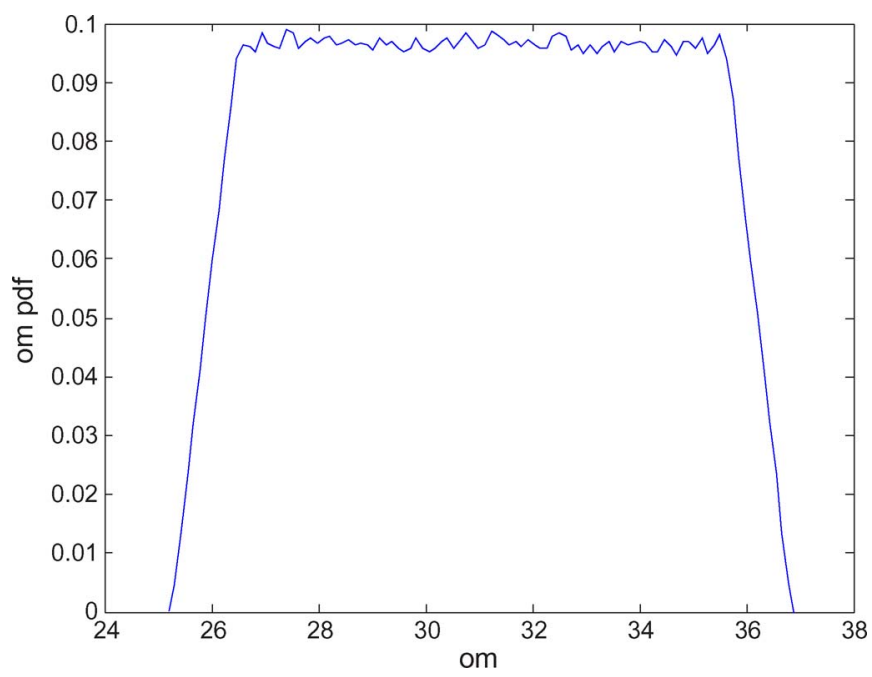

Fig. 7. PDF of the speed at $t=0.52$ interpreting the PCT model with a full probabilistic approach.

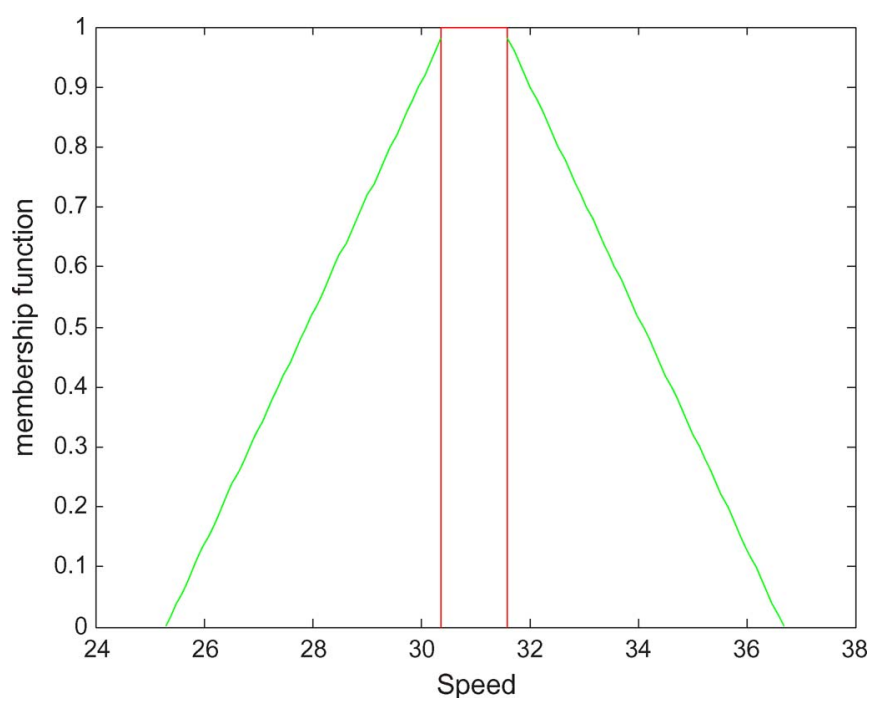

Fig. 8. RFV reconstruction under the same circumstances in Fig. 7.

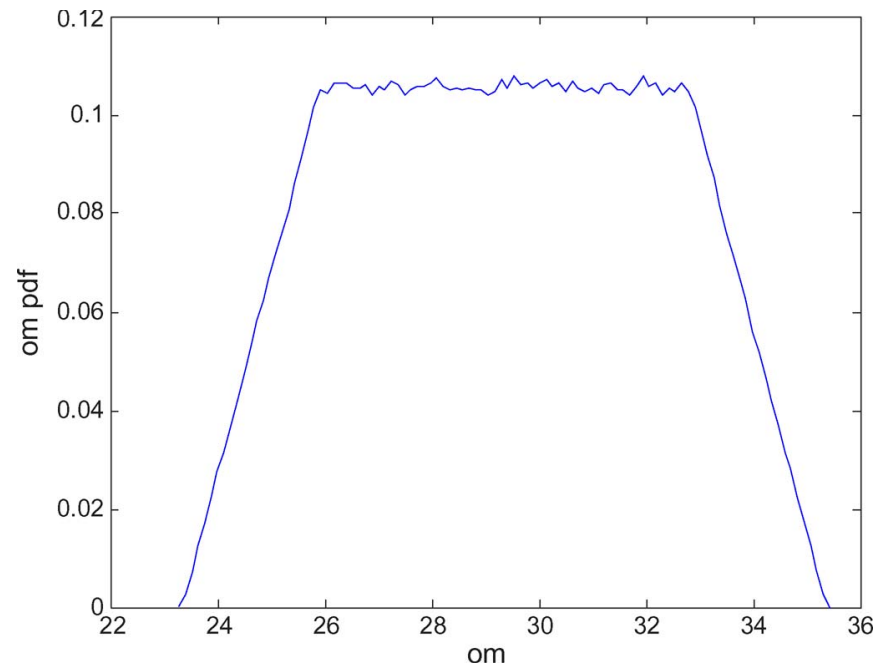

Fig. 9. PDF of the speed at $t=0.7$ interpreting the PCT model with a full probabilistic approach.

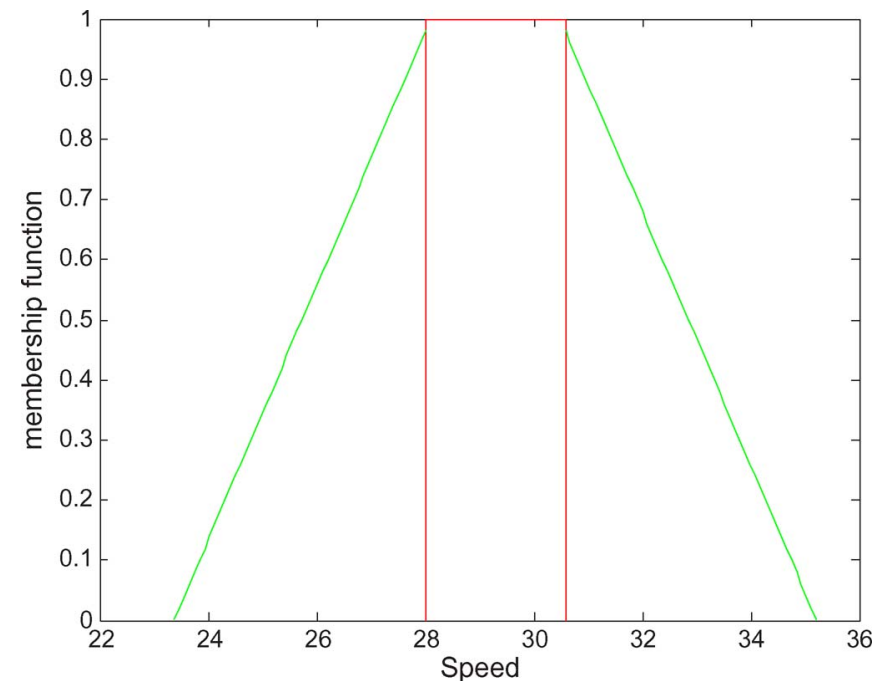

Fig. 10. RFV reconstruction under the same circumstances in Fig. 9.

Due to the approach proposed in this paper, the user can quickly evaluate the consequences of embracing one approach or the other and can quickly evaluate how the different approaches impact the confidence as a function of time.

\section{CONCLUSION}

This paper has introduced a new approach to the definition of polynomial chaos expansion of an uncertain variable in the presence of partly unknown uncertainty contributions. The goal to formalize a unified and flexible framework that can be used for processing uncertainties with known and unknown pdf has been achieved. The basic elements of algebra are introduced together with an example of application. The relation between this representation and the RFV approach is also introduced and investigated. A complete example is used for the demonstration of the process of variable combination and for the mapping to an equivalent RFV.

The major advantage of the proposed approach is that the user can compare the fully probabilistic approach with the 
random fuzzy variable and get an appreciation of the consequences of selecting one approach or the other.

While scientists are still divided over the philosophical implications of one approach or the other, the framework here proposed is able to capture in a single expression both results. In our opinion, the richness of the results gives the user the full power of understanding the consequences of accepting a specific interval for a specific coverage factor. Different applications may call for different approaches, depending also on the implications of the measurement under analysis.

One limitation of the proposed approach, currently, is to consider only two possible situations: 1) knowledge of the pdf of the uncertainty and 2) total ignorance of the distribution but knowledge of the interval of variation. The proposed framework is not able to capture the partial knowledge determined by systematic errors, as described, for example, in [16]. This aspect is still under investigation.

Furthermore, the analysis here reported can only be applied to single intervals and not to a set of nested intervals: this aspect is also still under investigation. What should appear clear is that PCT seems to be a very promising tool for simplifying the processing of the random part of an RFV variable.

\section{ACKNOWLEDGMENT}

The authors would like to thank Dr. A. Smith for his help in preparing the Maple script used for the PCT model expansion.

\section{REFERENCES}

[1] A. Monti, F. Ponci, and M. Valtorta, "Extending polynomial chaos to include interval analysis," in Proc. IEEE Workshop Adv. Methods Uncertainty Estimation Meas., Sardagna, Italy, Jul. 21-22, 2008, pp. 7-11.

[2] J. Y. Halpern, Reasoning About Uncertainty. Cambridge, MA: MIT Press, 2003

[3] G. Shafer, A Mathematical Theory of Evidence. Princeton, NJ: Princeton Univ. Press, 1976

[4] D. Xiu and G. E. Karniadakis, "The Wiener-Askey polynomial chaos for stochastic differential equations," SIAM J. Sci. Comput., vol. 24, no. 2, pp. 619-644, 2002.

[5] T. Lovett, A. Monti, and F. Ponci, "A polynomial chaos approach to measurement uncertainty," IEEE Trans. Instrum. Meas., vol. 55, no. 3, pp. 729-736, Jun. 2006.

[6] A. Smith, A. Monti, and F. Ponci, "Indirect measurements via polynomial chaos observer," IEEE Trans. Instrum. Meas., vol. 56, no. 3, pp. 743-748, Jun. 2007.

[7] G. D'Antona, A. Monti, F. Ponci, and L. Rocca, "Maximum entropy analytical solution for stochastic differential equations based on the Wiener-Askey polynomial chaos," IEEE Trans. Instrum. Meas., vol. 56, no. 3, pp. 689-695, Jun. 2007.

[8] A. Smith, A. Monti, and F. Ponci, "Robust controller using polynomial chaos theory," in Conf. Rec. IEEE IAS Annu. Meeting, Tampa, FL, Oct. 2006, pp. 2511-2517.

[9] A. Smith, A. Monti, and F. Ponci, "Uncertainty and worst-case analysis for a low pass filter using polynomial chaos theory," in Proc. IEEE AMUEM, Trento, Italy, Jul. 2007, pp. 59-63.

[10] A. Ferrero and S. Salicone, "Modeling and processing measurement uncertainty within the theory of evidence: Mathematics of random-fuzzy variables," IEEE Trans. Instrum. Meas., vol. 56, no. 3, pp. 704-716, Jun. 2007.

[11] A. Ferrero and S. Salicone, "An original fuzzy method for the comparison of measurement results represented as random-fuzzy variables," IEEE Trans. Instrum. Meas., vol. 56, no. 4, pp. 1292-1299, Aug. 2007.

[12] A. Monti, F. Ponci, T. Lovett, A. Smith, and R. Dougal, "Modeling of uncertainty and applications in monitoring and control of power electronics," in Proc. IEEE ACC, Portland, OR, 2005, pp. 2011-2016.

[13] L. Jaulin, M. Kieffer, O. Didrit, and E. Walter, Applied Interval Analysis. New York: Springer-Verlag, 2001
[14] A. Smith, A. Monti, and F. Ponci, "Confidence interval estimation starting from polynomial chaos expansion of uncertainty," in Proc. IEEE Workshop Adv. Method Uncertainty Estimation Meas., Sardagna, Italy, Jul. 21-22, 2008, pp. 12-16.

[15] J. Chiasson, Modeling and High Performance Control of Electric Machines. New York: Wiley-IEEE Press, 2005.

[16] A. Ferrero, R. Gamba, and S. Salicone, "A method based on random-fuzzy variables for online estimation of the measurement uncertainty of DSPbased instruments," IEEE Trans. Instrum. Meas., vol. 53, no. 5, pp. 1362 1369, Oct. 2004.

[17] A. H. C. Smith, A. Monti, and F. Ponci, "Uncertainty and worst-case analysis in electrical measurements using polynomial chaos theory," IEEE Trans. Instrum. Meas., vol. 58, no. 1, pp. 58-67, Jan. 2009.

[18] A. Ferrero and S. Salicone, "An innovative approach to the determination of uncertainty in measurements based on fuzzy variables," IEEE Trans. Instrum. Meas., vol. 52, no. 4, pp. 1174-1181, Aug. 2003.

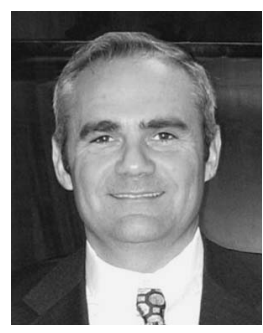

Antonello Monti (M'94-SM'02) received the M.S and $\mathrm{Ph} . \mathrm{D}$. degrees from "Politecnico di Milano," Milan, Italy, in 1989 and 1994, respectively, both in electrical engineering.

From 1990 to 1994 , he was with the research laboratory of Ansaldo Industria of Milan, where he was responsible for the design of the digital control of a large power cycloconverter drive. In 1995, he was an Assistant Professor with the Department of Electrical Engineering, "Politecnico di Milano." From 2000 to 2008, he was an Associate and then a Full Professor with the Department of Electrical Engineering, University of South Carolina, Columbia. He is currently the Director of the Institute for Automation of Complex Power Systems, E.ON Energy Research Center, Rheinisch-Westfaelische Technische Hochschule, Aachen, Germany.

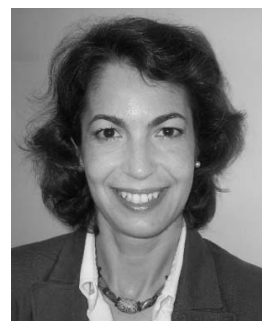

Ferdinanda Ponci (SM'08) received the M.S. and $\mathrm{Ph} . \mathrm{D}$. degrees from Politecnico di Milano, Milan, Italy, in 1998 and 2002, respectively, both in electrical engineering.

In 2003, she was with the Department of Electrical Engineering, University of South Carolina, Columbia, where she was an Assistant Professor with the Power and Energy Research Group and then promoted to Associate Professor in December 2008. She is currently with the Institute for Automation of Complex Power Systems, E.ON Research Center, Rheinisch-Westfaelische Technische Hochschule, Aachen University, Aachen, Germany. Her research is in methods for uncertainty representation and propagation and in multiagent systems for control and monitoring of power electronic systems.

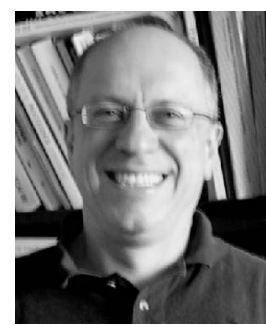

Marco Valtorta (SM'05) received the Laurea degree in electrical engineering from Politecnico di Milano, Milan, Italy, in 1980, and the M.A. and $\mathrm{Ph} . \mathrm{D}$. degrees in computer science from Duke University, Durham, NC, in 1984 and 1987, respectively.

Between 1985 and 1988, he was a Project Officer with ESPRIT at the Commission of the European Communities, Brussels, Germany, where he supervised projects in the advanced information processing area. Since 1988, he has been a faculty member with the University of South Carolina, Columbia, where he is currently a Professor of Computer Science and Engineering. He has conducted research funded by IARPA, SPAWAR, ARDA, DARPA, the Office of Naval Research (ONR), the U.S. Department of Agriculture (DOA), CISE (an Italian laboratory controlled by ENEL, the state electricity company), and the South Carolina Law Enforcement Division. He was an Associate Editor of the International Journal of Approximate Reasoning from 1993 to 2008 and is a member of the editorial board of Applied Intelligence. His research interests are in the area of normative reasoning under uncertainty (particularly Bayesian networks, influence diagrams, and their use in stand-alone and multiagent systems) and in other subfields of artificial intelligence. 